\title{
Concussion Leading to Parkinsonism and Debilitating Holmes Tremor in a Teenager, Case Report
}

\author{
${ }^{1}$ Geetanjali S Rathore, M.D, ${ }^{2}$ Shyamaly Premaraj, ${ }^{3}$ Paul D Larsen, M.D \\ 1Department of Pediatric Neurology, University of Nebraska Medical Center, Omaha, Nebraska, U S A \\ 2College of Medicine, University of Nebraska Medical Center, Omaha, Nebraska, U S A \\ 3Department of Pediatric Neurology, University of Nebraska Medical Center, Omaha, Nebraska, U S A
}

*Corresponding Author: Geetanjali S Rathore, Department of Pediatric Neurology, University of Nebraska Medical Centre, Omaha, Nebraska, U S A. E-mail; grathore@unmc.edu

\begin{abstract}
Post-traumatic movement disorders are seen in about $10 \%$ of patients following traumatic brain injury (TBI). These are mostly associated with severe TBI. Tremor is the most common movement disorder following TBI; it can be transient or follow a more persistent course. In most patients evidence of diffuse axonal injury is seen on magnetic resonance imaging. In contrast to tremor, Parkinsonism is very infrequently reported following acute TBI.

Injury to the dentato-rubro-thalmaic pathways and substantia nigra are postulated to be the underlying pathophysiology for post traumatic tremors and Parkinsonism, respectively. Pharmacological treatment in these patients is usually unsatisfactory.

A seventeen-year-old previously healthy male presented to our neurology clinic for chronic headaches following a concussion. Six months later he suffered another concussion due to fall from a tractor. He had no loss of consciousness, but within the next couple of hours developed severe tremor making him wheelchair dependent. On exam a coarse, 3-4 hz tremor was noted. Since it was predominantly in arms at rest and got much more exaggerated in lower extremities on trying to stand, it was concerning for Holmes tremor, typically seen in lesions of the midbrain. His stooped, slow shuffling gait and slight rigidity in the extremities was consistent with features of parkinsonism, which would also indicate a midbrain lesion. His MRI brain however was normal with no evidence of structural injury. He was treated with low midazolam and had significant improvement within 2 weeks with only a mild tremor residual in bilateral arms. The patient's severe manifestations following a relatively mild TBI, 'suggests a consequence of second impact syndrome, as he was still recovering from his prior concussion. This case re-enforces the debilitating consequences of concussion and the serious nature of second impact syndrome.
\end{abstract}

Keywords: Concussion, Traumatic brain injury, Holmes tremor, Parkinsonism, Second, impact syndrome

\section{INTRODUCTION}

Traumatic brain injuries (TBI) are very common, an estimated 1.74 million people sustaining one every year in USA. Mild TBI resulting from blunt force, non-penetrating head traumas frequently occur in contact sports. American football and soccer are the leading sports, where an estimated 20 percent of US high school and 10 percent of US college players sustain brain injuries every season [1]. Of the potential short and long-term sequelae following a TBI, post-traumatic movement disorders are seen in about $10 \%$ of patients. Tremor is the most common movement disorder, and can be transient or follow a more persistent course. Diffuse axonal injury seen on magnetic resonance imaging in patients with post-traumatic movement disorders has been postulated to play a role in mild TBI [4].

While tremors are a common post-traumatic movement disorder following a mild TBI, Parkinsonism, a condition causing a combination of movement abnormalities seen in Parkinson's disease, is very infrequently reported. Here we present a unique case of a seventeen-year-old previously healthy male who experienced debilitating Holmes tremor and Parkinsonism following a concussion. 


\section{CASE PRESEnTATiON}

A seventeen-year-old male initially presented to our neurology clinic for evaluation of chronic headachesfollowing a concussion with no loss of consciousness (LOC) sustained during football 3months ago. At primary care office he was diagnosed with a concussion and prescribed Fioricet (acetaminophen, butalbital, and caffeine) and Ketorolac. After a couple of doses of these he developed episodes of back arching and twitching of face, but no LOC or involvement of arms and legs. Both medications were stopped and the symptoms resolved, indicating a potential acute dystonic reaction to one of the drugs.The patient was normal upon physical examination except for slight difficulty with balance.Past medical, surgical and family history were not significant. CT/MRI brain and electroencephalogram (EEG) were normal. Creatinine kinase $(\mathrm{CK})$ and urine drug screen checked during initial emergency room visit for abnormal dystonic movements were unremarkable.The patient was advised to continue taking physical and mental rest and was started on amitriptyline, a headache prophylactic to help reduce the frequency of headaches.

The patient was seen 6 months later in the clinic, following a second concussive injury due to a fall from a tractor. Within an hour of the fall, the patient experienced a body tremor, which seemed to worsen when tired and attempting to walk. There was no LOC, vision changes, changes in cognition/speech, focal weakness, sensory changes, or bladder bowel dysfunction. Upon examination, the patient was in a wheelchair and in slight distress from the body tremor. He was alert, oriented with normal cognition. Speech had a slight tremor, no aphasia or dysarthria. Cranial nerves and reflexes were intact. On motor exam the patient had slight rigidity in extremities and noted to havea large amplitude, 3-4 Htz, non-rhythmic tremor of the head and upper body when sitting in a chair. On standing, the legs became involved as well. The patient attempted to take a few steps but had a stooped posture and was only able to shuffle. A MRI of the brain with and without contrast showed no evidence of structural injury to explain the presentation. The patient was prescribed diazepam and physical therapy as tolerated. The patient experienced an improvement over the next two weeks with complete resolution of symptoms at his one month follow up.

\section{DISCUSSION}

It is important to understand the various sequelae that follow mild TBI due to their frequency and range in severity. Of the posttraumatic movement disorders following TBI, tremor is most commonly seen. The majority of patients that experience posttraumatic tremor have a history of deceleration trauma, which points to a diffuse axonal injury- this conclusion is supported by neuroradiological findings. While conventional imaging may be normal, this type of axonal injury can be seen using diffusor tensor imaging (DTI) [4]. Our patient demonstrated a Holmes tremor, which is coarse and low amplitude, and is a combination of rest, postural, and kinetic tremors of the extremities. In this type of tremor, lesions of the thalamus and cerebellum or tracts such as the dento-rubro-thalamic tract (DRTT), involved in fine motor skills, have been noted [4]. In a study of 19 patients with both bilateral and unilateral posttraumatic kinetic tremors, 25 instances of tremor were accounted for, and lesions of the DRTT were found in 22 of the 25 instances [5]. Astudy using DTI on a patient who suffered head trauma and displayed intention and resting tremors and ataxic gait showed a thinning of the left DRTT. In the study, conventional brain MRI of the patient did not reveal a specific lesion, indicating that the injury of the left DRTT in the patient was a result of traumatic axonal injury. One study reconstructed the cerebello-thalamic and thalamo-cortical portions of the DRTT in six patients with TBIs such as ischemia and hemorrhage and found injury of the cerebellothalamic portion of the DRTT in three patients [3]. This study demonstrates injury to the DRTT in patients with TBI.

While posttraumatic tremor has been well document, Parkinsonism following an acute TBI is very infrequently reported. Generally, parkinsonian syndromes are mostly seen in boxers, where there is repeated head trauma. This form of Parkinsonism is called pugilistic Parkinsonism (PP), and is a chronic encephalopathy that results from the combined effects of subclinical concussions secondary to rotational acceleration traumas resulting from direct blows to the head. Usually, PP has a delayed presentation, and takes several years to manifest after the end of an active boxing career. Injury to the substantia nigra has been demonstrated in these cases by clinical trials using DaT scans [4]. In one study, a high definition computed tomography scan of the 
midbrain of a 37-year old man showed lowdensity lesions in the region of the substantia nigra, an area also indicated in Parkinson's disease [6]. Animal studies have also shown that mild TBI can result in neuroinflammation and dopamine dysregulation in the substantia nigra and in rats, it has been shown that TBI have the potential to induce progressive degeneration of nigrostriatal dopaminergic neurons [2]. This type of degeneration leads to Parkinson's like symptoms.

Our patient's experience of Parkinsonism is unique not only in that it occurred after a mild TBI and with no history of repeated head trauma, but also because it manifested within hours of the injury. The severity of symptoms experienced by our patient could be attributed to second impact syndrome, a term used to describe diffuse cerebral swelling that occurs after a second concussion while the patient is still symptomatic from an initial concussion [1]. In the first phase of second impact syndrome, patients typically suffer a mild concussive head injury that results in cerebral autoregulatory failure. Cerebral autoregulation refers to the ability of the arteries to uniformly dilate or constrict to keep cerebral flow constant. Evidence shows that TBI can induce problems with cerebral autoregulation for brief periods such as hours or days and in severe cases, weeks. The second injury can cause catecholamine release, rapid blood pressure elevation, and massive brain injury [7]. It is possible that our patient experienced the consequences of second impact syndrome due to the fact that he had an initial concussion from which he was still recovering when he received a second injury to the head. While the first injury did not produce severe symptoms, the second injury resulted in a greater range of symptoms, indicating that second impact syndrome may have played a role in the exacerbation of symptoms due to cerebral autoregulatory failure and a catecholamine rush.

\section{Conclusion}

Concussions are very common especially during recreational accidents and contact sports such as football and soccer [1]. Movement disorders can be sequelae in addition to cognitive, behavioral and other motor dysfunction.Even minor TBI can cause significant disability, especially if they are recurrent without recovery. As seen in our patient, concussion can lead to not only debilitating tremor but also Parkinsonism. Parkinsonism is not always from chronic TBI but also a possible result of second impact syndrome. Future research with more functional imaging such as Diffuser tensor imaging (DTI) and DaT scans will help us understand the mechanism for this better and hopefully identify patients at risk for more significant sequelae, preventing such severe disabilities.

\section{REFERENCES}

[1] Evans, R. W. (2015, April 29). Concussion and mild traumatic brain injury. Retrieved June 14, 2016, from http://www-uptodatecom.library1.unmc.edu:2048/contents/concussio n-and-mild-traumatic-brain-

injury?source=search_result\&search=concussion \&selectedTitle $=1 \sim 49$

[2] Formisano, R., \& Zasler, N. D. (2014, July/August). Posttraumatic Parkinsonism. Journal of Head Trauma Rehabilitation,29(4), 387-390. doi:10.1097/htr.0000000000000027

[3] Jang, S. H., \& Kwon, H. G. (2015). Injury of the dentato-rubro-thalamic tract in a patient with mild traumatic brain injury. Brain Injury,29(1314), $1725-1728$. doi:10.3109/02699052.2015.1075170

[4] Krauss, J. K., \& Jankovic, J. (2002, May). Head Injury and Posttraumatic Movement Disorders. Neurosurgery, 50(5), doi:10.1227/00006123-200205000-00003

[5] Krauss J. K., Wakhloo AK, Nobbe F, Trankle R, Mundinger F, Seeger W: MR pathological correlations of severe posttraumatic tremor. Neurol Res 17: 409-416, 1995.

[6] Nayernouri, T. (1985, September). Posttraumatic Parkinsonism. Surgical Neurology,24(3), 263264. doi:10.1016/0090-3019(85)90035-7

[7] Wetjen, N. M., Pichelmann, M. A., \& Atkinson, J. L. (2010, October). Second Impact Syndrome: Concussion and Second Injury Brain Complications. Journal of the American College of Surgeons,211(4), 553-557. doi:10.1016/j.jamcollsurg.2010.05.020

Citation: Geetanjali S Rathore, Shyamaly Premaraj \& Paul D Larsen. Concussion Leading to Parkinsonism and Debilitating Holmes Tremor in a Teenager, Case Report. ARC Journal of Neuroscience.2017; 2(2):4-6. doi:dx.doi.org/10.20431/2456-057X.0202002.

Copyright: (C) 2017 Authors. This is an open-access article distributed under the terms of the Creative Commons Attribution License, which permits unrestricted use, distribution, and reproduction in any medium, provided the original author and source are credited. 